\title{
Aposematism and the Handicap Principle
}

\author{
Øistein Haugsten Holen* and Thomas Owens Svennungsen
}

Centre for Ecological and Evolutionary Synthesis, Department of Biology, University of Oslo, P.O. Box 1066 Blindern, N-0316 Oslo, Norway

Submitted February 3, 2012; Accepted June 26, 2012; Electronically published September 27, 2012

Online enhancement: appendix.

\begin{abstract}
Aposematic prey use conspicuous warning signals to advertise their defenses to predators. It has long been recognized that the efficiency of a warning signal may be reduced if poorly defended prey (automimics) are present in the population. The handicap principle suggests that the use of warning signals by poorly defended prey may be kept in check if signaling is costly. Three mechanisms that involve signal costs have been proposed to facilitate honest warning signals in prey: go-slow behavior in predators, resource allocation trade-offs, and costs of detection alone. We study all three in a unified game-theoretical framework. We find that the go-slow mechanism and the resource allocation mechanism can introduce differential benefits and differential costs of signaling, respectively, and can support honest signaling in accordance with the handicap principle. When honest signaling is maintained by the goslow mechanism, conspicuous prey will necessarily suffer more attacks on average than cryptic prey. In contrast, when honest signaling is maintained by the resource allocation mechanism, cryptic prey will suffer more attacks. The detection cost mechanism lacks differential costs and benefits, and its potential for maintaining honest signaling equilibria is limited. We relate our results to intra- and interspecific correlations between conspicuousness and defense.
\end{abstract}

Keywords: honest signal, warning coloration, conspicuousness, secondary defense, automimicry, taste-rejection, pooling equilibrium.

\section{Introduction}

Much attention has been devoted to understanding how animal signals can remain honest when there are conflicting interests between signalers and signal receivers (reviewed in Maynard Smith and Harper 2003). A frequently discussed example is that of prey that signal the strength of their antipredatory defenses (e.g., escape abilities) to predators in order to deter attack (Nur and Hasson 1984; Grafen 1990; Vega-Redondo and Hasson 1993; Yachi 1995; Johnstone 1997; Bergstrom and Lachmann 2001; Maynard Smith and Harper 2003). Although honest signaling of defense strength might benefit the well-defended prey in a population and their potential predators, less defended

* Corresponding author; e-mail: o.h.holen@bio.uio.no.

Am. Nat. 2012. Vol. 180, pp. 629-641. (C) 2012 by The University of Chicago. 0003-0147/2012/18005-53628\$15.00. All rights reserved. DOI: $10.1086 / 667890$ prey that "cheat" by using the same signals as the welldefended prey may be favored by natural selection, thus rendering the signal unreliable. The handicap principle (Zahavi 1975; Grafen 1990), which states that signals can be kept honest if they are costly, provides one solution to this problem. Honest signaling of defense can be evolutionarily stable if the signal advertising strong defense incurs greater costs on weakly defended prey than on strongly defended prey (differential costs) or, alternatively, if it provides lower benefits for weakly defended prey than for strongly defended prey (differential benefits; Johnstone 1997).

The theoretical models that investigate honest signaling by prey have focused mainly on pursuit-deterrent signals of limited duration (Nur and Hasson 1984; Vega-Redondo and Hasson 1993; Bergstrom and Lachmann 2001). Such signals are emitted by prey in direct response to predatory threat, and include behaviors like stotting in ungulates and postures and alarm calls that indicate to the predator that it has been detected (Ruxton et al. 2004; Caro 2005). Differential costs of signaling arise easily for pursuit deterrence signals, because the time or energy spent on signaling in the presence of the predator reduces the quality that varies and is advertised (the ability to escape).

Aposematic prey use permanent warning signals (conspicuous coloration) to advertise the presence of antipredatory defenses such as toxins, spines, and stings (reviewed in Ruxton et al. 2004). Until recently, the handicap principle has had little impact on the theory of aposematism (but see Guilford and Dawkins 1993; Gamberale-Stille and Guilford 2003). This is not due to any shortcomings of the handicap principle itself: the model by Yachi (1995) could for instance be reinterpreted as a handicap model of aposematism, and it shows that honesty requires differential costs of signaling. The difficulty has instead been to provide plausible mechanisms for why warning signals should have differential costs (or benefits) in the first place (Guilford and Dawkins 1993; Ruxton et al. 2004). Recently, the interest for the question of whether warning signals may be honest indicators of defense has greatly increased: positive relationships between warning color intensity and 
toxicity has been observed within species (Bezzerides et al. 2007; Blount et al. 2012; Maan and Cummings 2012; Vidal-Cordero et al. 2012) and between species (Summers and Clough 2001; Cortesi and Cheney 2010), and several theoretical models have been developed to understand this phenomenon (Blount et al. 2009; Speed et al. 2010; Lee et al. 2011).

We evaluate three mechanisms that could potentially lead to honest warning signals. The first is based on the idea that predators "go slow" and are more cautious when approaching and handling prey types that are likely to be harmful (Guilford 1994). Such go-slow behavior can facilitate discrimination between undefended and defended prey (Guilford 1994). If conspicuous prey tend to be more toxic, for instance, predators may adopt more restrictive acceptance thresholds when tasting conspicuous prey. This will decrease the probability that the predator erroneously ingests undesirable prey and increase the probability that conspicuous prey survive through taste-rejection (Wiklund and Järvi 1982; Sillén-Tullberg 1985; Lindquist and Hay 1996; Williams et al. 2010). Go-slow behavior can also be adaptive in cases where prey use defenses that take effect before ingestion: many predators take special precautions to circumvent defensive stings, bites, or secretions of dangerous prey (O'Connell and Formanowicz 1998; Jackson et al. 2002; Eisner et al. 2005), and such carefulness may also increase prey survival. If the benefit to prey of go-slow behavior in predators outweighs the costs of increased detectability for well-defended prey but not for weakly defended prey, the use of conspicuous signals by the latter might be kept in check (Guilford 1994).

The second mechanism concerns resource allocation trade-offs in prey. If the same limiting resource is needed to produce both the warning signal and the defense, the signal will use up some of the defensive quality that it advertises, much in the same way as pursuit-deterrent signals may use up escape ability (Guilford and Dawkins 1993). Variable access to the resource may then result in differential costs of signaling. This mechanism was at first deemed unlikely, since no suitable candidate resource was known (Guilford and Dawkins 1993). The idea was rejuvenated by Blount et al. (2009), who proposed that pigments used in prey signals might play such a dual role since their antioxidant properties may also prevent selfdamage when storing toxins. Using a simulation model, they explored how prey should divide their resources between a signal and a defense depending on the amount of resources available to them. They found that signal conspicuousness and defense level could be positively or negatively related within a population at evolutionary equilibrium, depending on the range of resource levels present, and it was concluded that warning signals could be honest in accordance with the handicap principle
(Blount et al. 2009). This conclusion does not follow from their results, however, since the predators in their model do not use the level of display to infer the quality of prey before deciding whether or not to attack. Such inference on behalf of signal receivers is an essential feature of the theory of honest signaling (Grafen 1990; Maynard Smith and Harper 2003). Predator inference was accounted for in a more recent model of the resource allocation hypothesis (Lee et al. 2011). In this model, however, prey conspicuousness was in addition assumed to have a positive effect on fecundity, and the ensuing correlations between conspicuousness and toxicity were reported as qualitatively the same regardless of whether the predators used the signals to infer prey quality or not (Lee et al. 2011). It is therefore still an open question whether a resource allocation trade-off between signal and defense can maintain honest warning signals by itself.

The third mechanism we investigate concerns the detection costs of conspicuousness. Although such costs are also incorporated in the two first mechanisms, the idea here is that increased probability of detection by predators might in itself suffice to maintain honest signaling (Guilford and Dawkins 1993; Speed et al. 2010). Since weakly defended prey have a lower chance of surviving attack they may simply not afford the increased detectability associated with the conspicuous signals of better-defended prey. Using a simulation model, Speed et al. (2010) explored which signals prey should use depending on their environmentally determined defense level. Their simulations terminated in evolutionary equilibria that were honest on average, in the sense that prey using more conspicuous signals had higher mean levels of toxins than prey using less conspicuous signals, which suggested that detection costs alone may stabilize honest signaling.

Earlier treatments of the three mechanisms have been verbal or simulation based (Guilford and Dawkins 1993; Guilford 1994; Blount et al. 2009; Speed et al. 2010) and have treated the predator side only implicitly (i.e., no payoff structure has been assumed for the predator). In this article, we analyze the three mechanisms in a joint framework using simple arguments from evolutionary game theory. We identify necessary properties of honest signaling equilibria and find that honest signaling equilibria will have defining features that are characteristic for the mechanisms that maintain them, which can be explored empirically. We demonstrate that handicap signaling equilibria are feasible for two of the mechanisms by constructing complete examples with a specified predator payoff structure.

\section{The Model}

We consider a population of prey that exhibit a permanent, nonreversible warning signal. Individuals within the pop- 
ulation differ with respect to some physiological state $M$ that is environmentally determined early in life (e.g., by the larval host plant). The state of every individual is drawn from the same probability distribution, and prey develop their warning signal conditional on their allotted state. The signal trait is denoted $X$. (See table 1 for summary of notation.)

Each prey may encounter multiple predators during its lifetime. Predator encounters can be divided into three sequential stages (e.g., Leimar et al. 1986): on encounter, a predator must first detect the prey, then decide whether or not to attack it, and finally pursue, capture, and ingest it. The probability that a prey is killed in any one encounter can be formally written as $H=C A(1-S)$, where $C$ is the probability of detection (our measure of conspicuousness), $A$ is the probability of being attacked if detected, and $S$ is the probability of surviving if attacked. We assume that there is always a nonzero probability of being killed in an encounter, that is, that $C>0, A>0$, and $S<1$. As will be detailed below, $C, A$, and $S$ may all depend on the signal trait of the prey individual (i.e., on $X$ ). Moreover, $S$ will depend on the state $M$.

We make the standard assumptions that each prey encounters predators randomly at the same rate, that prey surviving an encounter with a predator are left completely unharmed (Svennungsen and Holen 2007; Blount et al. 2009; Speed et al. 2010) and that the warning signal does not interfere with functions of the prey organism outside the context of avoiding predation (Sillén-Tullberg and Bryant 1983; Blount et al. 2009; Speed et al. 2010; but see
Holen and Johnstone 2004; Lindstedt et al. 2009). Each prey must survive predation for a unit period of time before reproducing and dying. The predators search randomly and independently for prey, and the number of predator encounters an individual must survive in order to reproduce is Poisson distributed. Formally, the probability of surviving until reproduction for a prey that survives each encounter with probability $1-H$ is $F(H)=$ $e^{-n H}$, where $n$ is the average rate of predator encounters (a standard assumption; e.g., Svennungsen and Holen 2007; Blount et al 2009; Speed et al. 2010; Lee et al. 2011). Thus, natural selection on the signaling strategy acts so as to minimize $H$. It immediately follows that conspicuous signals cannot be advantageous unless they either reduce the probability of being attacked after detection (by being more aversive; Sillén-Tullberg and Bryant 1983) or increase the probability of surviving attack (e.g., via go-slow behavior; Guilford 1994).

The prey strategies are probabilistic: for each possible prey state, they specify a probability distribution from which the signal is drawn. The prey strategy may thus put positive probability on more than one signal in each state (mixing). In addition, prey of different states may put positive probability on the same signal (pooling, sensu Bergstrom and Lachmann 1998). We assume that there are at least two different signals available to the prey and that there are at least two different states. However, we need not specify whether the signal trait or the state distribution is continuous or discrete; it makes no difference to the analysis.

Table 1: Summary of notation

\begin{tabular}{ll}
\hline Symbol & Explanation \\
\hline$X$ & Signal trait \\
$M$ & Prey state \\
$H$ & Probability of being killed in an encounter \\
$C$ & Probability of detection (conspicuousness) \\
$A$ & Probability of attack given detection \\
$S$ & Probability of surviving attack \\
$n$ & Average rate of predator encounters \\
$p_{k}$ & Probability of signaling $X_{1}$ in state $M_{k}$ \\
$q$ & Fraction of prey in state $M_{1}$ \\
$q^{\prime}$ & Posterior probability (after visual inspection) that a prey is in state $M_{1}$ \\
$q^{\prime \prime}$ & Posterior probability (after taste sampling) that a prey is in state $M_{1}$ \\
$f_{i k}$ & Fraction of prey with signal $X_{i}$ and state $M_{k}$ \\
$f_{i k}^{\prime}$ & Posterior probability (after visual inspection) that a prey is in state $M_{k}$ \\
$T$ & $\quad$ and with signal $X_{i}$ \\
$B$ & Prey bitterness \\
$U$ & Expected benefit of attacking a prey \\
$K$ & Payoff from consuming a captured prey \\
$z$ & Cost of attacking a prey \\
$Z$ & Standard normal density function \\
\hline
\end{tabular}


At an honest signaling equilibrium, the signals must be at least partly informative about the strength of the prey defenses so that the predators benefit from using the signals to infer prey quality before deciding whether or not to attack and consume prey. In other words, the signals must be honest "on average" (Johnstone and Grafen 1993). We will investigate honest signaling in a two-step fashion. In the first section, we focus on the prey side and identify necessary conditions for honest signaling equilibria to exist (cf. Grafen 1990; Bowles and Hammerstein 2003). These conditions are our main results and can be used to determine whether a given mechanism for honest signaling is consistent with empirical observations. In the second section, we proceed to model the predator side in more detail. We verify that two of the mechanisms can support honest signaling equilibria and show that the third is unlikely to do so.

\section{Evolutionary Stability of Prey Signaling}

We start out by supposing that a signaling equilibrium that is honest has already been reached: a conditional signaling strategy that specifies the distribution of signals to use in the different states is fixed in the prey population, while a receiver strategy that determines how different signals are treated is fixed in the predator population; both strategies are evolutionarily stable given the strategy played by the other (Riechert and Hammerstein 1983).

The probability of detection (conspicuousness) for a signal is given by some strictly increasing function, $C(X)$. Let $A^{*}(X)$ denote the probability that a predator will attack a prey with signal $X$ on detection at evolutionary equilibrium. The function $A^{*}(X)$ is determined by the evolutionarily stable strategy (ESS) of the predators. Here, and in the following, the asterisk serves as a reminder that the function depends on predators' response to the signal and refers to values at evolutionary equilibrium. Let $S^{*}(X, M)$ denote the probability that a prey in state $M$ with signal $X$ will survive attack at evolutionary equilibrium. As will be clear, in the go-slow scenario, $S^{*}(X, M)$ will partly depend on the ESS strategy of the predators; in the other scenarios, $S^{*}(X, M)$ does not depend on how predators interpret signals and is thus independent of the predator strategy. (The asterisk on $S$ is thus superfluous in the two latter scenarios and is retained only to keep notation consistent across all three scenarios.)

At an honest signaling equilibrium, at least two different signals must be in use in the prey population. We can therefore pick two different signals that are used at the equilibrium and label them $X_{i}$ and $X_{j}$, where by convention $X_{i}>X_{j}$. Let $M_{k}$ denote a state for which the evolutionary stable strategy puts positive probability on developing signal $X_{i}$, and let $M_{l}$ denote a state for which it puts positive probability on developing signal $X_{j}$. The labels $M_{k}$ and $M_{i}$ might refer to two different states or to the same state (in the case of a mixed strategy). Given that the conditional signaling strategy is evolutionarily stable, it follows from the first part of the ESS definition (Maynard Smith 1982) that

$$
\begin{aligned}
& \exp \left[-n C\left(X_{i}\right) A^{*}\left(X_{i}\right)\left(1-S^{*}\left(X_{i}, M_{k}\right)\right)\right] \\
& \geq \exp \left[-n C(X) A^{*}(X)\left(1-S^{*}\left(X, M_{k}\right)\right)\right]
\end{aligned}
$$

must hold for all $X$. This inequality simply says that at the signaling equilibrium, switching to a signal different from $X_{i}$ when in state $M_{k}$ will not lead to a higher payoff. It follows from equation (1a) that

$$
\begin{aligned}
& C\left(X_{i}\right) A^{*}\left(X_{i}\right)\left(1-S^{*}\left(X_{i}, M_{k}\right)\right) \\
\leq & C\left(X_{j}\right) A^{*}\left(X_{j}\right)\left(1-S^{*}\left(X_{j}, M_{k}\right)\right) .
\end{aligned}
$$

Applying the same reasoning to state $M_{l}$ yields

$$
\begin{aligned}
& C\left(X_{j}\right) A^{*}\left(X_{j}\right)\left(1-S^{*}\left(X_{j}, M_{l}\right)\right) \\
\leq & C\left(X_{i}\right) A^{*}\left(X_{i}\right)\left(1-S^{*}\left(X_{i}, M_{l}\right)\right) .
\end{aligned}
$$

The three scenarios are defined by different assumptions about $S^{*}(X, M)$. In the following sections we present and combine these assumptions with inequalities (1b) and (1c), $X_{i}>X_{j}$, and $C\left(X_{i}\right)>C\left(X_{j}\right)$ and derive necessary conditions for honest signaling under each mechanism. Since we have made no assumptions about the signals labeled $X_{i}$ and $X_{j}$ other than that they are present at the equilibrium and satisfy $X_{i}>X_{j}$, and no assumptions about the states labeled $M_{k}$ and $M_{l}$, equivalent conditions can be derived for any pair of signals present at the equilibrium.

The Go-Slow Scenario. In this scenario, the environmentally determined state of an individual, $M$, refers directly to its defense level (e.g., toxin level determined by the larval host plant). Signals can be produced at no physiological cost. Two key assumptions are made about the function $S^{*}(X, M)$. First, we assume that $S^{*}(X, M)$ increases in its second argument, reflecting that the probability of surviving attack increases with the strength of the defense. Second, we assume that $S^{*}\left(X_{i}, M\right)-$ $S^{*}\left(X_{j}, M\right)$ has the same sign as $A^{*}\left(X_{j}\right)-A^{*}\left(X_{i}\right)$ for all $M$. This simply reflects that the predators go slow and exhibit different levels of caution in response to different signals at equilibrium: the signal that is less likely to elicit attack from predators (once detected) also elicits greater caution if approached and handled. (In the second section, we show that this assumption represents optimal behavior.) A prey that is attacked by a more cautious predator is more likely to survive attack.

Since $C\left(X_{i}\right)>C\left(X_{j}\right)$, the expressions $S^{*}\left(X_{i}, M_{k}\right)-$ $S^{*}\left(X_{j}, M_{k}\right)$ and $A^{*}\left(X_{j}\right)-A^{*}\left(X_{i}\right)$ cannot both be negative 
or both be zero at the honest signaling equilibrium (this would violate inequality [1b]). The only possibility left is that they are both positive. This implies $A^{*}\left(X_{i}\right)<A^{*}\left(X_{j}\right)$ and that $S^{*}\left(X_{i}, M_{l}\right)-S^{*}\left(X_{j}, M_{l}\right)$ is also positive. Inequality (1c) can then hold only if $C\left(X_{j}\right) A^{*}\left(X_{j}\right)<C\left(X_{i}\right) A^{*}\left(X_{i}\right)$.

The Resource Allocation Scenario. In this scenario, the environmentally determined state of an individual, $M$, refers to a resource level. The resource can be allocated toward a conspicuous signal or a secondary defense, and has no other use (Guilford and Dawkins 1993; Blount et al. 2009). The prey signaling strategy is in effect an allocation rule: it prescribes, for each state, how to partition the resource between signal and defense. The trade-off between survival and conspicuousness is embodied in the probability of surviving attack for different signals $X$ when in some resource state $M$, that is, $S^{*}(X, M)$ (fig. 1$)$. Two key assumptions are made about the function $S^{*}(X, M)$. First, $S^{*}(X, M)$ is strictly decreasing in its first argument (until it reaches zero), reflecting that increased investment in signal conspicuousness reduces the resources available for defense. Second, $S^{*}(X, M)$ is strictly increasing in the second argument, reflecting that more resources leaves more for defense for the same level of conspicuousness. Note that prey in a low-resource state may be constrained from producing the more costly signal, so inequality (1c) may not necessarily apply.

From $X_{i}>X_{j}$ and the fact that $S^{*}(X, M)$ decreases in the first argument, it follows that $S^{*}\left(X_{i}, M_{k}\right)<S^{*}\left(X_{j}, M_{k}\right)$. Inequality (1b) can then hold only if $C\left(X_{i}\right) A^{*}\left(X_{i}\right)<$ $C\left(X_{j}\right) A^{*}\left(X_{j}\right)$, which together with $C\left(X_{i}\right)>C\left(X_{j}\right)$ implies $A^{*}\left(X_{i}\right)<A^{*}\left(X_{j}\right)$.

The Detection Cost Scenario. In this scenario, $M$ again refers directly to defense level. Two key assumptions are made about the function $S^{*}(X, M)$. First, $S^{*}(X, M)$ increases in its second argument, reflecting that the probability of surviving attack increases with the defense level. Second, the probability of surviving if attacked is independent of the signal and uniquely defined by the defense state (i.e., $S^{*}\left(X_{i}, M\right)=S^{*}\left(X_{j}, M\right)$ for all $\left.M\right)$. The only cost of signaling is in terms of increased probability of detection, and this cost is independent of state. Since $S^{*}\left(X_{i}, M_{k}\right)=S^{*}\left(X_{i}, M_{k}\right)$ and $S^{*}\left(X_{i}, M_{l}\right)=S^{*}\left(X_{j}, M_{l}\right)$, inequalities (1b) and (1c) can both hold only if they both hold weakly. This in turn implies that

$$
C\left(X_{j}\right) A^{*}\left(X_{j}\right)=C\left(X_{i}\right) A^{*}\left(X_{i}\right) .
$$

Since $C\left(X_{i}\right)>C\left(X_{j}\right)$, equality (2) implies $A^{*}\left(X_{j}\right)>$ $A^{*}\left(X_{i}\right)$. This latter result is consistent with the findings of Sillén-Tullberg and Bryant (1983): when the probability of surviving attack is independent of the signal, a con-

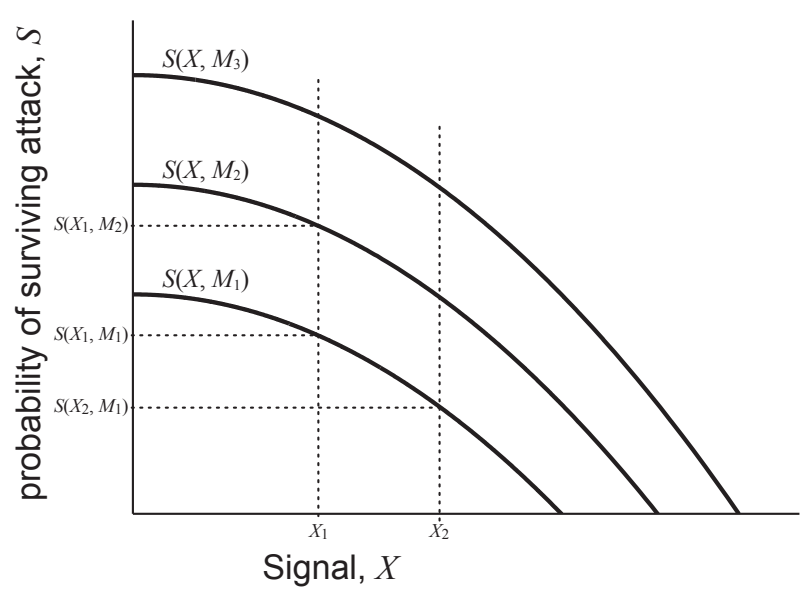

Figure 1: The resource allocation hypothesis: possible trade-offs between conspicuousness and the probability of surviving attack is shown for three different resource states $M_{1}, M_{2}$, and $M_{3}$, satisfying $M_{1}<M_{2}<M_{3}$. Diverting more resources into the signal increases conspicuousness and reduces the efficiency of the defense. Clearly, if $X_{1}<X_{2}$, it must be the case that $S^{*}\left(X_{1}, M_{1}\right)<S^{*}\left(X_{1}, M_{2}\right)$ and $S^{*}\left(X_{1}, M_{1}\right)>S^{*}\left(X_{2}, M_{1}\right)$ (the latter assuming that $X_{2}$ can actually be produced when in resource state $\left.M_{1}\right)$.

spicuous signal can only be favored by natural selection if it is more aversive than a cryptic one.

Necessary Conditions for Honest Warning Signals. In all three scenarios, we find that honest signaling is possible only if the predators are more reluctant to attack the more conspicuous signal on detection (i.e., that $A^{*}\left(X_{j}\right)>$ $\left.A^{*}\left(X_{i}\right)\right)$. In other words, the conspicuousness of a signal (i.e., probability of detection) must be inversely related to the probability that a predator will attack it on detection. Clearly, if the predators act optimally and can perfectly discriminate between signals, they will not be more prone to attack signals associated with a low expected payoff than signals associated with a high expected payoff. At equilibrium, the prey using a conspicuous signal must therefore be less profitable to the predator (on average) than those using a less conspicuous one. A positive relationship between conspicuousness and defense is therefore expected. This does not mean that the evolutionarily stable signaling equilibrium needs to be perfectly honest with a one-toone correspondence between state and signal, but the signals must convey enough information for the predator to treat them differently. In other words, the signaling equilibrium must be honest "on average" (Johnstone and Grafen 1993).

If the predators cannot perfectly discriminate between the signals, signaling equilibria may arise in which prey with certain conspicuous signals are more profitable (on average) to the predator than less conspicuous prey. For 
instance, a rare prey type with a weak defense could potentially benefit from signaling at a level of conspicuousness intermediate to the levels of two better-defended and more common prey types, given that it was sufficiently often misclassified as being one of these common types. Averaged across all prey, however, the conspicuousness of prey and the expected payoff from attacking them must nevertheless be negatively related, or the predators would not maintain their aversion for conspicuous signals (cf. Johnstone and Grafen 1993). An overall positive relationship between conspicuousness and defense is therefore expected.

The overall attack rate on a signal is determined not only by the predators' willingness to attack it after detection but also by how easy the signal is to detect. We define the "probability of attack on encounter" as the probability of detection times the probability of attack after detection (i.e., the product $C(X) A^{*}(X)$ ). In the go-slow scenario, an honest signaling equilibrium is possible only if conspicuous prey are attacked more often on encounter than inconspicuous prey at equilibrium (i.e., $C\left(X_{j}\right) A^{*}\left(X_{j}\right)<$ $\left.C\left(X_{i}\right) A^{*}\left(X_{i}\right)\right)$. In contrast, in the resource allocation scenario, honest signaling is possible only if conspicuous prey are less likely to be attacked on encounter than inconspicuous prey (i.e., $\left.C\left(X_{j}\right) A^{*}\left(X_{j}\right)>C\left(X_{i}\right) A^{*}\left(X_{i}\right)\right)$. In the detection cost scenario, honest signaling is only possible if all signals are equally likely to be attacked on encounter.

Further necessary conditions for honest signaling can be found by combining inequalities (1b) and (1c) to obtain

$$
\frac{1-S^{*}\left(X_{j}, M_{l}\right)}{1-S^{*}\left(X_{i}, M_{l}\right)} \leq \frac{C\left(X_{i}\right) A^{*}\left(X_{i}\right)}{C\left(X_{j}\right) A^{*}\left(X_{j}\right)} \leq \frac{1-S^{*}\left(X_{j}, M_{k}\right)}{1-S^{*}\left(X_{i}, M_{k}\right)},
$$

which implies

$$
\begin{aligned}
& \ln \left(1-S^{*}\left(X_{i}, M_{k}\right)\right)-\ln \left(1-S^{*}\left(X_{j}, M_{k}\right)\right) \\
\leq & \ln \left(1-S^{*}\left(X_{i}, M_{l}\right)\right)-\ln \left(1-S^{*}\left(X_{j}, M_{l}\right)\right) .
\end{aligned}
$$

At equilibrium, a switch from the less conspicuous signal $\left(X_{j}\right)$ to the more conspicuous signal $\left(X_{i}\right)$ cannot lead to a higher reduction (or a lower increase) in the logged probability of being killed on attack for the prey in state $M_{l}$ than the prey in state $M_{k}$.

When inequality (4) holds strictly, it encapsulates the differential costs or benefits that are present at handicap equilibria: in the go-slow scenario, less conspicuous prey do not benefit from using the signals of more conspicuous prey because they would obtain a smaller decrease (on a log scale) in the probability of being killed on attack than the latter (differential benefits). In the resource allocation scenario, less conspicuous prey do not benefit from using the signals of more conspicuous prey because they would suffer a greater increase (on a log scale) in the probability of being killed on attack than the latter (differential costs).
The use of more conspicuous signals by weakly defended prey than better defended prey (cheating) can be stable only if the weakly defended prey enjoy lower differential costs (or greater differential benefits) of signaling. The detection cost scenario lacks differential costs or benefits, since in this case inequality (4) holds only weakly.

Innate versus Learned Predator Preferences. The predator behavior at equilibrium might represent innate preferences or may be the outcome of learning. In the latter case, we may think of the evolutionarily stable predator strategy as a genetically based rule or recipe that describes the process by which the predator learns about the prey. The results above are derived under the assumption that all predators express the same preferences at evolutionary equilibrium, which is reasonable if the preferences are innate or, in the case of learning, if the learning period is short relative to predator life span and the population consists almost entirely of fully educated predators that behave close to the optimum.

When learning is a more time-consuming process, the behavioral variation among predators at evolutionary equilibrium is likely to be non-negligible. Such variation makes no difference to the resource allocation and the detection cost scenario: we may simply replace the monomorphic attack probabilities $A^{*}\left(X_{i}\right)$ and $A^{*}\left(X_{j}\right)$ in inequalities (1a)-(1c) with the corresponding mean attack probabilities and proceed to derive essentially the same results. In the go-slow scenario, however, the probability of surviving attack $(S)$ is also influenced by the predator strategy. Suppose that the distribution of learning states in the predator population reaches a steady state at evolutionary equilibrium. The probability that a prey with signal $X_{i}$ and state $M_{k}$ survives an encounter with a predator can then be written as

$$
\begin{aligned}
H & =C\left(X_{i}\right) \mathrm{E}\left[A_{i}\left(1-S_{i k}\right)\right] \\
& =C\left(X_{i}\right) \mathrm{E}\left[A_{i}\right]\left(1-\mathrm{E}\left[S_{i k}\right]\right)-C\left(X_{i}\right) \operatorname{Cov}\left[A_{i}, S_{i k}\right],
\end{aligned}
$$

where $A_{i}$ and $S_{i k}$ are stochastic variables that represent the probabilities of attack upon detection and the probabilities of surviving attack which vary across predators according to the joint equilibrium distribution. Since $H$ here contains a covariance term, the results obtained for the go-slow scenario cannot be reformulated in terms of mean attack and survival probabilities.

When predator preferences are innate (i.e., genetically hard-wired), an additional result emerges. In this case, it is necessary for the evolutionary stability of the prey strategy that inequality (1a) holds strictly for all $X \neq X_{i}$. This means that the prey strategy cannot be mixed. To see that this must be the case, note that if inequality (1a) held weakly for some signal $X^{\prime} \neq X_{i}$, a mutant prey strategy 
that put slightly higher probability on developing signal $X^{\prime}$ and slightly lower probability on $X_{i}$ when in state $M_{k}$ but was otherwise equal to the resident strategy could invade by neutral drift. An increase in the frequency of such a mutant could be counteracted only by an evolutionary change in the predator preferences, and the signaling equilibrium would therefore not be stable. (This result does not apply when preferences are learned, since predators may then respond to changes in the prey population on a faster behavioral timescale and potentially in such a way that mutant prey will be selected against.) From the requirement that inequality (1a) must hold strictly, it follows that inequalities (1b), (1c), (3), and (4) must also hold strictly. This rules out the detection cost mechanism as an explanation for honest signaling when preferences are innate.

\section{Evolutionarily Stable Predator Strategies}

In this section it will be demonstrated that equilibria that satisfy the necessary conditions for honest signaling are feasible. In the go-slow scenario and the resource allocation scenario, we construct examples of honest signaling equilibria in which predator and prey strategies are unique best replies to each other. For the detection cost scenario, we will argue that honest signaling equilibria (if at all possible) are biologically unlikely.

For simplicity, we consider only two prey states, $M_{1}$ and $M_{2}$, and a signal trait with only two possible values, $X_{1}$ and $X_{2}$, where (by convention) $X_{1}<X_{2}$. The detection probabilities are $C\left(X_{1}\right)$ and $C\left(X_{2}\right)$, respectively, where $C\left(X_{1}\right)<C\left(X_{2}\right)$. The prey strategies are vectors $\left[p_{1}, p_{2}\right]$, with elements $p_{1}$ and $p_{2}$ specifying the probabilities by which to develop signal $X_{1}$ in states $M_{1}$ and $M_{2}$, respectively. (Signal $X_{2}$ is developed with probabilities $1-p_{1}$ and $1-p_{2}$, respectively.) The fractions of prey in states $M_{1}$ and $M_{2}$ at equilibrium are $q$ and $(1-q)$, where $0<q<1$.

Construction of Examples. We assume that predators assess a prey's signal on detection, but that cognitive constraints and environmental noise prevents perfect perception of the signal trait. Perceptual error serves to maintain a positive attack probability for both signals at equilibrium. The predator strategy prescribes for each possible perceived signal value whether the prey should be attacked or ignored. In the go-slow scenario, the predators taste-sample captured prey, and the predator strategy in addition prescribes for each possible combination of perceived signal and perceived taste whether a captured prey should be ingested or rejected. The attack and pursuit of a prey incurs a cost $K$ to the predator in terms of time and energy that could have been spent on other activities. We assume that the optimal predator strategy is the one that maximizes expected payoff per prey encounter (Johnstone 2002; Sherratt 2002b; Holen and Johnstone 2004). At the ESS, a predator will only attack a prey if the expected benefit from attacking it outweighs the cost $K$.

Formally, inaccurate perception is incorporated by letting perceived trait value be drawn from a normal distribution centered on the true value with unit standard deviation. Suppose that the prey population is fixed for some strategy $\left[\hat{p}_{1}, \hat{p}_{2}\right]$. Let $f_{i k}\left(\hat{p}_{1}, \hat{p}_{2}\right)$ denote the fraction of the prey that are in state $M_{k}$ with signal $X_{i}$; we have $f_{11}\left(\hat{p}_{1}, \hat{p}_{2}\right)=\hat{p}_{1} q, \quad f_{21}\left(\hat{p}_{1}, \hat{p}_{2}\right)=\left(1-\hat{p}_{1}\right) q, \quad f_{12}\left(\hat{p}_{1}, \hat{p}_{2}\right)=$ $\hat{p}_{2}(1-q)$, and $f_{22}\left(\hat{p}_{1}, \hat{p}_{2}\right)=\left(1-\hat{p}_{2}\right)(1-q)$. If a prey is perceived to have signal value $X$, the posterior probability that it is in state $M_{k}$ with signal $X_{i}$ is

$$
\begin{gathered}
f_{i k}^{\prime}\left(X, \hat{p}_{1}, \hat{p}_{2}\right)= \\
\frac{f_{i k} C\left(X_{i}\right) z\left(X-X_{i}\right)}{\left(f_{21}+f_{22}\right) C\left(X_{2}\right) z\left(X-X_{2}\right)+\left(f_{11}+f_{12}\right) C\left(X_{1}\right) z\left(X-X_{1}\right)},
\end{gathered}
$$

where $z(\cdot)$ is the probability density of the standard normal distribution. This represents the best estimate a predator can have of the probability of prey identity when deciding whether or not to attack a prey. Let $q^{\prime}\left(X, \hat{p}_{1}, \hat{p}_{2}\right)$ denote the posterior probability that the prey is in state $M_{1}$, that is,

$$
q^{\prime}\left(X, \hat{p}_{1}, \hat{p}_{2}\right)=f_{11}^{\prime}\left(X, \hat{p}_{1}, \hat{p}_{2}\right)+f_{21}^{\prime}\left(X, \hat{p}_{1}, \hat{p}_{2}\right) .
$$

At signaling equilibrium, the predator should attack a prey if the expected benefit of doing so is greater than the cost. The benefit of attack will be derived separately for each scenario below.

The Go-Slow Scenario. In this scenario, the environmentally determined state of a prey is a level of defense, which we assume is a distasteful toxin. The amount of toxin affects predator payoff: let $U_{1}$ and $U_{2}$, where $U_{1}>K>$ $0>U_{2}$, denote the payoff obtained from consuming a captured prey in state $M_{1}$ and $M_{2}$, respectively. The payoff is zero if the predator releases the prey. For simplicity, the probability of prey capture is assumed to be the same for all prey and set to unity to reduce notational load. Predators carefully taste-sample the prey they catch, and we let the "bitterness" of prey in state $M_{1}$ and $M_{2}$, respectively, be represented by $T_{1}$ and $T_{2}$, where $T_{1}<T_{2}$. The bitterness perceived by the predator is assumed drawn from a normal distribution with unit standard deviation, centered on the true value of bitterness. This assumption is consistent with studies of taste discrimination (Irwin et al. 1992).

The optimal attack strategy can be identified through backward induction, that is, by first determining the optimal taste-sampling strategy and thereby the expected benefit of attack. At the time of capture the probability 
that the prey is in state $M_{1}$ is given by $q^{\prime}$ (eq. [6b]). For a perceived bitterness $T$, the updated posterior probability that the captured prey is in state $M_{1}$ is

$$
q^{\prime \prime}(T)=\frac{q^{\prime} z\left(T-T_{1}\right)}{q^{\prime} z\left(T-T_{1}\right)+\left(1-q^{\prime}\right) z\left(T-T_{2}\right)} .
$$

The expected payoff for ingesting a prey with perceived bitterness $T$ is thus $q^{\prime \prime}(T) U_{1}+\left(1-q^{\prime \prime}(T)\right) U_{2}$. The predator should ingest the prey whenever this expression is positive and release it otherwise. The optimal rejection threshold for bitterness, $T^{*}$, found by solving $q^{\prime \prime}(T) U_{1}+(1-$ $\left.q^{\prime \prime}(T)\right) U_{2}=0$ for $T$, is

$$
T^{*}\left(q^{\prime}\right)=\frac{T_{1}+T_{2}}{2}-\frac{\ln \left[\left(1-q^{\prime}\right)\left(-U_{2}\right) /\left(q^{\prime} U_{1}\right)\right]}{T_{2}-T_{1}} .
$$

The optimal taste-sampling strategy is to ingest prey perceived as less bitter than this threshold. Importantly, the taste rejection threshold depends on the perceived signal: the function $T^{*}\left(q^{\prime}\right)$ strictly increases with $q^{\prime}$, which means that the predator should adopt a stricter (lower) acceptance threshold for bitterness when it is more certain that the prey is in state $M_{2}$ (fig. 2). Go-slow behavior thus emerges naturally from the model assumptions.

When the predator uses the optimal taste-sampling strategy, the expected benefit of attacking a prey with signal perceived as $X$ is

$$
B\left(X, \hat{p}_{1}, \hat{p}_{2}\right)=V\left(q^{\prime}\left(X, \hat{p}_{1}, \hat{p}_{2}\right)\right),
$$

where

$$
\begin{aligned}
V\left(q^{\prime}\right)= & q^{\prime} Z\left(T^{*}\left(q^{\prime}\right)-T_{1}\right) U_{1} \\
& +\left(1-q^{\prime}\right) Z\left(T^{*}\left(q^{\prime}\right)-T_{2}\right) U_{2},
\end{aligned}
$$

and $Z(\cdot)$ denotes the cumulative standard normal distribution function. If the prey signal differently in the two states (i.e., $\hat{p}_{1} \neq \hat{p}_{2}$ ), then $q^{\prime}$ is strictly monotonic in $X$, and since $V\left(q^{\prime}\right)$ is strictly increasing, $B$ is strictly monotonic in $X$.

Suppose now that prey always signal $X_{1}$ when in defense state $M_{1}$ and $X_{2}$ when in state $M_{2}$ (i.e., the prey population is fixed for strategy $[1,0])$. Since $B$ is monotonic in $X$, the predator should attack all prey with signals perceived as falling below the threshold $X^{*}$ (fig. 2), where $X^{*}$ is the unique solution to $B(X, 1,0)-K=0$. When predators use the optimal strategy, prey with signal $X_{i}$ will be attacked with probability

$$
A^{*}\left(X_{i}\right)=Z\left(X^{*}-X_{i}\right)
$$

on detection. This function is strictly decreasing; the predators are less inclined to attack the more defended prey at equilibrium. Assuming for simplicity that the probability of survival is unity for all prey that are tasted and

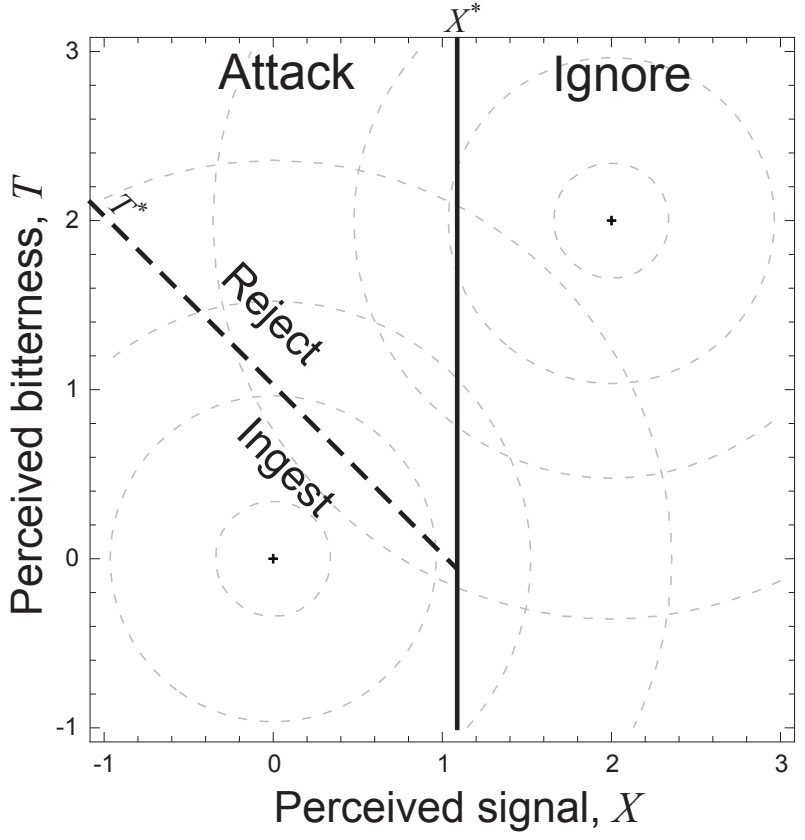

Figure 2: The optimal predator strategy in the go-slow scenario. Predators should attack prey only on detection if the perceived signal falls below the threshold $X^{*}$ (thick solid line). On capture, the predators should ingest only prey whose perceived bitterness falls below the flexible threshold $T^{*}$ (thick dashed line). The crosses indicate the true values of signal and bitterness for the two prey types. Due to perceptual noise, the perceived values of signal and bitterness are binormally distributed (the gray dashed lines show contours of the density distributions of perceived prey characteristics). See main text for parameter values.

subsequently rejected, the probability that a prey in state $M_{1}$ that has developed signal $X_{i}$ will survive an attack is

$$
\begin{gathered}
S^{*}\left(X_{i}, M_{1}\right)= \\
1-\int_{-\infty}^{X^{*}} \frac{z\left(X-X_{i}\right)}{Z\left(X^{*}-X_{i}\right)} Z\left[T^{*}\left(q^{\prime}\left(X, \hat{p}_{1}, \hat{p}_{2}\right)\right)-T_{1}\right] d X .
\end{gathered}
$$

(To calculate survival probability in state $M_{2}$, replace $T_{1}$ with $T_{2}$.) We can choose the following parameter values: $q=1 / 2, K=1 / 10, U_{1}=3, U_{2}=-3, X_{1}=0, X_{2}=2$, $T_{1}=0, T_{2}=2, C\left(X_{1}\right)=1 / 8, C\left(X_{2}\right)=7 / 8$. Using equations (6a), (6b), (8), (9a), and (9b), the optimal attack threshold can be calculated numerically as $X^{*}=1.088$. Using equations (10) and (11), we obtain $A^{*}\left(X_{1}\right)=$ 0.862, $\quad A^{*}\left(X_{2}\right)=0.181, \quad S^{*}\left(X_{1}, M_{1}\right)=0.160$, $S^{*}\left(X_{1}, M_{2}\right)=0.716, \quad S^{*}\left(X_{2}, M_{1}\right)=0.334, \quad$ and $S^{*}\left(X_{2}, M_{2}\right)=0.915$. These values satisfy the strict inequalities 


$$
\begin{aligned}
& C\left(X_{2}\right) A^{*}\left(X_{2}\right)\left(1-S^{*}\left(X_{2}, M_{2}\right)\right) \\
& <C\left(X_{1}\right) A^{*}\left(X_{1}\right)\left(1-S^{*}\left(X_{1}, M_{2}\right)\right)
\end{aligned}
$$

and

$$
\begin{aligned}
& C\left(X_{1}\right) A^{*}\left(X_{1}\right)\left(1-S^{*}\left(X_{1}, M_{1}\right)\right) \\
< & C\left(X_{2}\right) A^{*}\left(X_{2}\right)\left(1-S^{*}\left(X_{2}, M_{1}\right)\right) .
\end{aligned}
$$

Thus, by first providing a model of predator inference resulting in go-slow behavior, we have calculated the (strictly) optimal predator strategy given the prey strategy $[1,0]$ and in turn shown that the prey strategy $[1,0]$ is (strictly) optimal given the optimal predator strategy. We have verified that the go-slow mechanism can support a handicap signaling equilibrium.

Resource Allocation Scenario. In this scenario the state of prey corresponds to an amount of resource that can be allocated between a signal and a defense. The predator strategy prescribes for each possible perceived signal value whether the predator should attack, but it does not affect the probability that prey survives attack. For simplicity, we assume that the prey will either survive an attack or be consumed by the predator. Letting $M_{1}<M_{2}$, the assumptions of the resource allocation scenario require that $S^{*}\left(X_{1}, M_{1}\right)>S^{*}\left(X_{2}, M_{1}\right), \quad S^{*}\left(X_{1}, M_{2}\right)>S^{*}\left(X_{2}, M_{2}\right)$, $S^{*}\left(X_{1}, M_{1}\right)<S^{*}\left(X_{1}, M_{2}\right)$, and $S^{*}\left(X_{2}, M_{1}\right)<S^{*}\left(X_{2}, M_{2}\right)$ (fig. 1). Predators obtain the payoff $U\left(X_{i}, M_{k}\right)$ if they consume a prey in resource state $M_{k}$ with signal $X_{i}$. The expected payoff to a predator that attacks such a prey is $(1-$ $\left.S^{*}\left(X_{i}, M_{k}\right)\right) U\left(X_{i}, M_{k}\right)$. The expected benefit of attacking a prey with signal perceived as $X$ is

$$
\begin{gathered}
B\left(X, \hat{p}_{1}, \hat{p}_{2}\right)= \\
\sum_{i=1}^{2} \sum_{k=1}^{2} f_{i k}^{\prime}\left(X, \hat{p}_{1}, \hat{p}_{2}\right) U\left(X_{i}, M_{k}\right)\left(1-S^{*}\left(X_{i}, M_{k}\right)\right) .
\end{gathered}
$$

Suppose again that prey always signal $X_{1}$ when in defense state $M_{1}$ and $X_{2}$ when in state $M_{2}$ (i.e., the prey population is fixed for strategy $[1,0])$. The expected benefit of attacking a prey then simplifies to

$$
B(X, 1,0)=L\left(q^{\prime}(X, 1,0)\right),
$$

where

$$
\begin{aligned}
L\left(q^{\prime}\right)= & q^{\prime} U\left(X_{1}, M_{1}\right)\left(1-S^{*}\left(X_{1}, M_{1}\right)\right) \\
& +\left(1-q^{\prime}\right) U\left(X_{2}, M_{2}\right)\left(1-S^{*}\left(X_{2}, M_{2}\right)\right) .
\end{aligned}
$$

We focus on the nontrivial case in which predators benefit from attacking prey in state 1 and avoiding those in state 2, that is, where $U\left(X_{1}, M_{1}\right)\left(1-S^{*}\left(X_{1}, M_{1}\right)\right)>K>$ $U\left(X_{2}, M_{2}\right)\left(1-S^{*}\left(X_{2}, M_{2}\right)\right)$. This ensures that $L\left(q^{\prime}\right)$ is strictly increasing. As before, the optimal predator strategy given the prey strategy $[1,0]$ is to adopt a threshold, $X^{*}$, which is the unique solution to $B(X, 1,0)-K=0$, and attack all prey with signals perceived as falling below the threshold.

Our illustrative example involves a toxic defense. We assume that the predators tend to taste-reject defended prey, but that their propensity to do so is unaffected by the perceived signal (i.e., predators do not go slow). The payoff to the predator when consuming a prey is inversely related to the prey's probability of surviving attack; formally, $U\left(X_{1}, M_{1}\right)<U\left(X_{2}, M_{1}\right), U\left(X_{1}, M_{2}\right)<U\left(X_{2}, M_{2}\right)$, $U\left(X_{1}, M_{1}\right)>U\left(X_{1}, M_{2}\right)$, and $U\left(X_{2}, M_{1}\right)>U\left(X_{2}, M_{2}\right)$. We choose the following parameter values for our example: $q=1 / 2, \quad K=1 / 10, \quad X_{1}=0, \quad X_{2}=2, \quad C\left(X_{1}\right)=1 / 10$, $C\left(X_{2}\right)=9 / 10, \quad S^{*}\left(X_{1}, M_{1}\right)=3 / 10, \quad S^{*}\left(X_{2}, M_{1}\right)=1 / 10$, $S^{*}\left(X_{1}, M_{2}\right)=5 / 10, S^{*}\left(X_{2}, M_{2}\right)=4 / 10, U\left(X_{1}, M_{1}\right)=9 / 5$, and $U\left(X_{2}, M_{2}\right)=-1 / 2$. Using equations (6a), (6b), (14a), (14b), the optimal attack threshold can be calculated numerically as $X^{*}=0.434$. Using equation (10) we obtain $A^{*}\left(X_{1}\right)=0.668, A^{*}\left(X_{2}\right)=0.059$. These values satisfy the strict inequalities (12a), (12b). We have calculated the (strictly) optimal predator strategy given the prey strategy $[1,0]$ and in turn shown that the prey strategy $[1,0]$ is (strictly) optimal given the predator strategy. We conclude that the resource allocation mechanism can support a handicap signaling equilibrium.

The examples of signaling equilibria constructed above are perfectly honest with a one-to-one correspondence between signal and state. In appendix section 1, available online, we give an example of an evolutionarily stable pooling equilibrium that is not perfectly honest but honest "on average" (Johnstone and Grafen 1993): the equilibrium involves prey in an intermediate resource state that use the same conspicuous signal as the better-defended prey in the highest resource state. The prey in the intermediate resource state are "cheats" that are profitable to the predator but protected by their resemblance to the better defended unprofitable prey. In appendix section 2, we also provide an additional example of honest signaling in which the defense is harmless to the predator but aids escape; the defense might be evasive flight, startling behavior, or the ability to wrestle free from a predator's grasp.

The Detection Cost Scenario. As noted above, honest signaling equilibria in the detection cost scenario can be ruled out when predator preferences are innate. We have explored the detection cost scenario under the assumption that predator preferences change on a faster timescale due to optimal learning (appendix section 3). The model makes no assumptions with respect to learning mechanisms or predator psychology but does assume that the attack behavior of predators is at any point in time instantaneously determined by the state of the prey popu- 
lation (a rough but standard approximation; Johnstone 2002; Holen and Johnstone 2004; Broom et al. 2005; Svennungsen and Holen 2007; Blount et al. 2009; Speed et al. 2010; Lee et al. 2011). Imposing a lack of differential costs and benefits causes the model to display some unusual and degenerate features: there are infinitely many singular strategies (i.e., strategies for which the local fitness gradients are zero; Geritz et al. 1998), and they trace out curves in the $\left(p_{1}, p_{2}\right)$ plane instead of occurring as isolated points. The model also lacks structural robustness: adding an infinitesimal state-dependent cost of signaling causes all singular strategies to vanish altogether (appendix section 3). Although we have not explored this model with predator preferences changing on an intermediary timescale, it is likely that the lack of structural robustness will prevail; the singular prey strategies must vanish when the model is infinitesimally perturbed by state-dependent costs of signaling. We explain in appendix section 4 how the basic detection cost model by Speed et al. (2010; i.e., without their "recurrent perturbation" procedure) suffers from the same limitations.

\section{Discussion}

We find that go-slow behavior in predators (Guilford and Dawkins 1993; Guilford 1994) and resource allocation trade-offs in prey (Guilford and Dawkins 1993; Blount et al. 2009) can keep warning coloration honest in accordance with the handicap principle. The two mechanisms can give rise to differential benefits and costs of signaling, respectively. In both cases, we find that any honest signaling equilibrium must be such that the predator is more reluctant to attack conspicuous signals on detection (i.e., conspicuous signals must be more aversive than cryptic ones). This in turn suggests that the overall relationship between conspicuousness and defense must be positive. The detection cost scenario lacks differential costs and benefits of signaling; it cannot be interpreted as a handicap mechanism and is unlikely to support honest signaling equilibria.

The idea that go-slow behavior may maintain honest signaling was originally presented verbally (Guilford 1994); our treatment provides the first formal examination of this idea. The go-slow mechanism does not posit any direct physiological coupling between the defense and the signal; it can be classified as a handicap mechanism with receiverdependent costs and benefits (Guilford and Dawkins 1995). It could apply to a broad range of defenses. Our analysis clarifies some conditions that are absolutely necessary for honest signaling to be evolutionarily stable: the benefit of conspicuous signaling lies in the inducement of go-slow behavior in predators, which increases the probability of surviving attack (Guilford 1994). At an honest signaling equilibrium, prey that use conspicuous signals will suffer the cost of an increased probability of being attacked on encounter (i.e., an increase in the product of the probability of detection and the probability of attack given detection). The less conspicuous prey do not benefit from using more conspicuous signals because they would not obtain a sufficiently high increase in the probability of surviving attack. Guilford (1994) argued that conspicuous signals can act as go-slow signals only if there is something especially effective about them, for instance, if they allow for particularly fast caution learning in predators. Our results show that no such effect is necessary. It should be mentioned that Guilford's original treatment of go-slow behavior was more complex as it was also concerned with the stability of the secondary defense itself (Guilford 1994); we have not explored stability of defenses here (but see Svennungsen and Holen 2007). Our treatment is therefore most relevant for prey defenses that are largely determined by the environment, such as toxins that are sequestered from the larval host plant. It should also be noted that our results for the go-slow scenario are obtained under the assumption that there is little variation in predator behavior at equilibrium. If preferences are learned and the learning process is slow, the variation in behavior at equilibrium may be non-negligible. An explicit model of the learning process may be needed to study circumstances in detail.

Sherratt (2002a) has argued that the more conspicuous a prey item is, the more likely it is to be defended, for the simple reason that it is more likely to have been detected by other predators beforehand and survived. Predation thus weeds out the undefended conspicuous prey and gradually increases the average level of defense among the conspicuous prey. The same process occurs among cryptic prey but is slower due to lower detection rates. Although models have shown that this filtering process may cause predators to be more willing to attack cryptic prey than conspicuous prey, different conclusions have been drawn regarding its potential for driving the evolution of conspicuousness among defended prey (Sherratt 2002a; Franks and Noble 2004). Sherratt's idea is close in spirit to Zahavi's original formulations of the handicap principle, in which males with well-developed and costly sexual traits are attractive to females because these males have proven capable of surviving in spite of their costly traits (Zahavi 1975). The go-slow mechanism analyzed here can be considered a strategic choice handicap mechanism (sensu Grafen 1990) from which the essential feature of Sherratt's process emerges: since conspicuous prey suffer more attacks on average than cryptic prey at the honest signal equilibrium, a conspicuous prey is more likely than a cryptic prey of having been attacked by other predators beforehand and survived. Conspicuousness is thus an effec- 
tive signal that the carrier is well defended and is able to survive attacks, as proposed by Sherratt (2002a). The higher number of attacks on conspicuous prey is due to increased detection, since predators (at equilibrium) have a lower probability (given detection) of pursuing conspicuous prey than cryptic prey.

The characteristics of honest signaling equilibria in the resource allocation scenario are strikingly different from those in the go-slow scenario. Most importantly, the benefit of conspicuous signaling is now a reduced probability of attack on encounter, that is, a reduction in the product of the probability of detection and the probability of attack given detection. This is more in line with the standard explanations for the existence of conspicuous warning signals (e.g., facilitation of predator learning and enhanced memorability; Ruxton et al. 2004), which seem to imply that warning coloration ultimately functions to decrease the number of attacks on individual prey. The benefit of conspicuous signaling is independent of the signaler's resource level and requires only that the signal can be afforded in the first place. The cost of conspicuous signaling is a reduced defense level and thus a reduced probability of surviving when attacked, which does depend on the resource level available to the prey. At an honest signaling equilibrium, less conspicuous prey do not benefit from using more conspicuous signals because it would entail a too-high reduction in the probability of surviving attack. Our results for the resource allocation scenario seem incompatible with the negative relationship between conspicuousness and defense level found by Blount et al. (2009) for high resource availabilities. Their finding reflects their model assumption that conspicuousness acts as a defensive trait in its own right and not as a signal component by which defense level can be inferred.

Positive correlation between signal intensity and defense within a population has been found in two species of ladybird beetles (Bezzerides et al. 2007; Blount et al. 2012). Seven-spotted ladybird beetles (Coccinella septempuntata) reared on a limited diet have been shown to attain lower levels of a defensive alkaloid and a less intense red coloration than those reared on a plentiful diet, which has been interpreted as indicative of a resource-allocation trade-off (Blount et al. 2012). An alternative interpretation is that the diet constrains the concentration of the defensive alkaloid but not the signal and that the signal is developed conditionally on defense level as predicted by the go-slow hypothesis. Ladybird beetles have hard cuticles and reflex-bleed when handled, which suggests that they may survive taste-sampling without severe damage. If the go-slow hypothesis holds true, one should expect conspicuous individuals on average to suffer more attacks (but survive better) than cryptic prey under natural conditions. It would be of great interest to obtain relevant field data that would allow attack rates to be separated from mortality rates in order to test this prediction.

The detection cost mechanism does not involve differential costs or benefits and therefore cannot be considered a handicap mechanism. Evolutionary stability of honest signaling under the detection cost mechanism would require that all signals in use were equally effective at equilibrium (i.e., that the product of the probability of detection and the probability of attack given detection is exactly the same for all of them). This peculiar condition ensures that evolutionary stability of signaling equilibria is impossible when the predator preferences are innate. In addition, our analysis of a simple model with instantaneous predator learning reveals that all signaling equilibria with potential for evolutionary stability are knife-edge cases or lack structural robustness; they vanish when infinitesimal state-dependent costs to signaling are added to the model. The equilibria in the detection cost model of Speed et al. (2010) also lack structural robustness. This suggests to us that equilibrium solutions in the detection cost scenario are of little real-world importance. To better understand how signals evolve when differential costs are zero (or very small), it will be necessary to use a stochastic approach that allows for evolutionary drift. Nonequilibrium dynamics or nonsignaling equilibria are likely outcomes.

We must emphasize that it is not our view that most instances of aposematism should be honest. Aposematism may in many cases be a dynamical (transient) phenomenon that is vulnerable to destabilization by the appearance of conspicuous, undefended automimics. Our message is simply that under certain conditions, aposematism might also represent evolutionarily stable honest signaling equilibria, with conspicuous signals being beneficial only for sufficiently well-defended prey. Some limited cheating can nevertheless be present at an honest equilibrium: prey in different states may form pools that share the same signal, and some of the prey within a given signal pool may obtain better protection than they would have done if they had their own unique signal (Grafen 1990; Bergstrom and Lachmann 1998). When predators do not perceive signals perfectly, it may also be possible for sufficiently rare prey types with weak defenses to use levels of conspicuousness that are intermediate to the levels used by more common and better defended prey types.

Our results are also relevant for multiple populations or related species of aposematic prey that are exposed to the same population of predators. We may then think of each state as corresponding to a population-specific or species-specific defense or resource level, and the prey strategy within each population as being unconditional. Using such an interpretation, Batesian mimicry could represent a stable level of cheating existing within an honest multispecies signaling system (Grafen 1990; Maynard 
Smith and Harper 2003) instead of being a dynamic and destabilizing evolutionary phenomenon. We therefore disagree with the proposition that Batesian mimicry is not expected if warning signals are handicaps (Guilford and Dawkins 1993), which stemmed from an overly restrictive interpretation of the handicap principle that seemed to exclude many forms of receiver-dependent costs. We would also like to point out that even if the handicap principle does not require that the different signals should bear any resemblance to each other, such resemblance can be integral to many specific handicap mechanisms. In our go-slow example, for instance, it is imperfect discrimination (caused by signal resemblance) that maintains the positive attack probability on conspicuous prey and stabilizes the signaling system. In the resource allocation mechanism of Blount et al. (2009), the hypothesized resource is an antioxidant pigment. If a resource allocation trade-off involving such a pigment is common to a group of related species, their warning signals may involve different amounts of the pigment at the honest signaling equilibrium, but may not necessarily differ in pattern. Therefore, the argument that the widespread pattern resemblance among aposematic species is an indication that the handicap principle is less important for warning coloration (Guilford and Dawkins 1993) seems poorly founded. The mechanisms for honesty that we have explored suggest an overall positive relationship between conspicuousness and defense across populations or species, as observed in several studies (Summers and Clough 2001; Cortesi and Cheney 2010; Maan and Cummings 2012).

Observations of positive correlations between conspicuousness and defense within and between prey species do not in themselves constitute strong evidence for honest signaling. For example, it cannot be ruled out that conspicuousness could come to correlate with defense level for reasons other than predator deterrence, with the warning function arising as a secondary effect when predators start paying attention to conspicuousness (Lee et al. 2011). Nevertheless, our results have shown that go-slow behavior and resource allocation trade-offs are coherent and logically sound explanations for honest warning signals by prey. The possibility that warning signals are handicaps therefore deserves further empirical and theoretical study.

\section{Acknowledgments}

We thank B. Fischer, R. A. Johnstone, O. Leimar, T. Slagsvold, M. P. Speed, and the anonymous reviewers for comments on various versions of the manuscript and T. M. Jonassen and A. Seierstad for helpful discussions. Funding was provided by the Centre for Ecological and Evolutionary Synthesis, University of Oslo.

\section{Literature Cited}

Bergstrom, C. T., and M. Lachmann. 1998. Signaling among relatives. III. Talk is cheap. Proceedings of the National Academy of Sciences of the USA 95:5100-5105.

. 2001. Alarm calls as costly signals of antipredator vigilance: the watchful babbler game. Animal Behaviour 61:535-543.

Bezzerides, A. L., K. J. McGraw, R. S. Parker, and J. Husseini. 2007. Elytra color as a signal of chemical defense in the Asian ladybird beetle Harmonia axyridis. Behavioral Ecology and Sociobiology 61: 1401-1408.

Blount, J. D., H. M. Rowland, F. P. Drijfhout, J. A. Endler, R. Inger, J. J. Sloggett, G. D. D. Hurst, D. J. Hodgson, and M. P. Speed. 2012. How the ladybird got its spots: effects of resource limitation on the honesty of aposematic signals. Functional Ecology 26:334342 .

Blount, J. D., M. P. Speed, G. D. Ruxton, and P. A. Stephens. 2009. Warning displays may function as honest signals of toxicity. Proceedings of the Roval Society B: Biological Sciences 276:871-877.

Bowles, S., and P. Hammerstein. 2003. Does market theory apply to biology? Pages 153-165 in P. Hammerstein, ed. Genetic and cultural evolution of cooperation. MIT Press, Cambridge, MA.

Broom, M., M. P. Speed, and G. D. Ruxton. 2005. Evolutionarily stable investment in secondary defences. Functional Ecology 19: 836-843.

Caro, T. 2005. Antipredator defenses in birds and mammals. University of Chicago Press, Chicago.

Cortesi, F., and K. L. Cheney. 2010. Conspicuousness is correlated with toxicity in marine opistobranchs. Journal of Evolutionary Biology 23:1509-1518.

Eisner, T., M. Eisner, and D. Aneshansley. 2005. Pre-ingestive treatment of bombardier beetles by jays: food preparation by "anting" and "sand-wiping." Chemoecology 15:227-233.

Franks, D. W., and J. Noble. 2004. Warning signals and predatorprey coevolution. Proceedings of the Roval Society B: Biological Sciences 271:1859-1865.

Gamberale-Stille, G., and T. Guilford. 2003. Contrast versus colour in aposematic signals. Animal Behaviour 65:1021-1026.

Geritz, S. A. H., É. Kisdi, G. Meszéna, and J. A. J. Metz. 1998. Evolutionarily singular strategies and the adaptive growth and branching of the evolutionary tree. Evolutionary Ecology 12:3557.

Grafen, A. 1990. Biological signals as handicaps. Journal of Theoretical Biology 144:517-546.

Guilford, T. 1994. "Go-slow" signalling and the problem of automimicry. Journal of Theoretical Biology 170:311-316.

Guilford, T., and M. S. Dawkins. 1993. Are warning colors handicaps? Evolution 47:400-416.

- 1995. What are conventional signals? Animal Behaviour 49: $1689-1695$.

Holen, $\varnothing$. H., and R. A. Johnstone. 2004. The evolution of mimicry under constraints. American Naturalist 164:598-613.

Irwin, R. J., M. J. Hautus, and J. A. Stillman. 1992. Use of the receiver operating characteristic in the study of taste perception. $\underline{\text { Journal }}$ of Sensory Studies 7:291-314.

Jackson, R. R., S. D. Pollard, D. Li, and N. Fijn. 2002. Interpopulation 
variation in the risk-related decisions of Portia labiata, an araneophagic jumping spider (Aranea, Salticidae), during predatory sequences with spitting spiders. Animal Cognition 5:215-223.

Johnstone, R. A. 1997. The evolution of animal signals. Pages 155178 in J. R. Krebs and N. B. Davies, eds. Behavioural ecology: an evolutionary approach. Blackwell Science, Oxford.

- 2002. The evolution of inaccurate mimics. Nature 418:524526.

Johnstone, R. A., and A. Grafen. 1993. Dishonesty and the handicap principle. Animal Behaviour 46:759-764.

Lee, T. J., M. P. Speed, and P. A. Stephens. 2011. Honest signaling and the uses of prey coloration. American Naturalist 178:E1-E9.

Leimar, O., M. Enquist, and B. Sillén-Tullberg. 1986. Evolutionary stability of aposematic coloration and prey unprofitability: a theoretical analysis. American Naturalist 128:469-490.

Lindquist, N., and M. E. Hay. 1996. Palatability and chemical defense of marine invertebrate larvae. Ecological Monographs 66:431-450.

Lindstedt, C., L. Lindström, and J. Mappes. 2009. Thermoregulation constrains effective warning signal expression. Evolution 63:469478.

Maan, M. E., and M. E. Cummings. 2012. Poison frog colors are honest signals of toxicity, particularly for bird predators. American Naturalist 179:E1-E14.

Maynard Smith, J. 1982. Evolution and the theory of games. Cambridge University Press, Cambridge.

Maynard Smith, J., and D. Harper. 2003. Animal signals. Oxford University Press, Oxford.

Nur, N., and O. Hasson. 1984. Phenotypic plasticity and the handicap principle. Iournal of Theoretical Biology 110:275-297.

O'Connell, D. J., and D. R. Formanowicz Jr. 1998. Differential handling of dangerous and non-dangerous prey by naive and experienced Texas spotted whiptail lizards, Cnemidophorus gularis. Journal of Herpetology 32:75-79.

Riechert, S. E., and P. Hammerstein. 1983. Game theory in the ecological context. Annual Review of Ecology and Systematics 14:377409.

Ruxton, G. D., T. N. Sherratt, and M. P. Speed. 2004. Avoiding attack: the evolutionary ecology of crypsis, warning signals, and mimicry. Oxford University Press, Oxford.

Sherratt, T. N. 2002a. The coevolution of warning signals. Proceedings of the Roval Society B: Biological Sciences 269:741-746.
- 2002b. The evolution of imperfect mimicry. Behavioral Ecology 13:821-826.

Sillén-Tullberg, B. 1985. Higher survival of an aposematic than of a cryptic form of a distasteful bug. Oecologia (Berlin) 67:411-415.

Sillén-Tullberg, B., and E. H. Bryant. 1983. The evolution of aposematic coloration in distasteful prey: an individual selection model. Evolution 37:993-1000.

Speed, M. P., G. D. Ruxton, J. D. Blount, and P. A. Stephens. 2010. Diversification of honest signals in a predator-prey system. Ecology Letters 13:744-753.

Summers, K., and M. E. Clough. 2001. The evolution of coloration and toxicity in the poison frog family (Dendrobatidae). Proceedings of the National Academy of Sciences of the USA 98:62276232.

Svennungsen, T. O., and $\varnothing$. H. Holen. 2007. The evolutionary stability of automimicry. Proceedings of the Roval Society B: Biological Sciences 274:2055-2062.

Vega-Redondo, F., and O. Hasson. 1993. A game-theoretic model of predator-prey signaling. Lournal of Theoretical Biology 162:309319.

Vidal-Cordero, J. M., G. Moreno-Rueda, A. López-Orta, C. MarfilDaza, J. L. Ros-Santaella, and F. J. Ortiz-Sánchez. 2012. Brightercolored paper wasps (Polistes dominula) have larger poison glands. Frontiers in Zoology 9:20, doi:10.1186/1742-9994-9-20.

Wiklund, C., and T. Järvi. 1982. Survival of distasteful insects after being attacked by naive birds: a reappraisal of the theory of aposematic coloration evolving through individual selection. Evolution 36:998-1002.

Williams, B. L., C. T. Hanifin, E. D. Brodie Jr., and E. D. Brodie III. 2010. Tetrodoxin affects survival probability of rough-skinned newts (Taricha granulosa) faced with TTX-resistant garter snake predators (Thamnophis sirtalis). Chemoecology 20:285-290.

Yachi, S. 1995. How can honest signalling evolve? the role of handicap principle. Proceedings of the Roval Society B: Biological Sciences 262:283-288.

Zahavi, A. 1975. Mate selection: a selection for a handicap. Journal of Theoretical Biology 53:205-214.

Associate Editor: Thomas N. Sherratt Editor: Troy Day 